\title{
Massively Deployable, Low-Cost Airborne Sensor Motes for Atmospheric Characterization
}

\author{
Michael Bolt, J. Craig Prather, Tyler Horton, Mark Adams \\ Department of Electrical and Computer Engineering, Auburn University, Auburn, AL, USA \\ Email:mtb0026@auburn.edu
}

How to cite this paper: Bolt, M., Prather, J.C., Horton, T. and Adams, M. (2020) Massively Deployable, Low-Cost Airborne Sensor Motes for Atmospheric Characterization. Wireless Sensor Network, 12, 1-11. https://doi.org/10.4236/wsn.2020.121001

Received: January 2, 2020

Accepted: January 28, 2020

Published: January 31, 2020

Copyright (c) 2020 by author(s) and Scientific Research Publishing Inc. This work is licensed under the Creative Commons Attribution International License (CC BY 4.0).

http://creativecommons.org/licenses/by/4.0/

\begin{abstract}
A low-cost airborne sensor mote has been designed for deployment en masse to characterize atmospheric conditions. The designed environmental sensing mote, or eMote, was inspired by the natural shape of auto-rotating maple seeds to fall slowly and gather data along its descent. The eMotes measure and transmit temperature, air pressure, relative humidity, and wind speed estimates alongside GPS coordinates and timestamps. Up to 2080 eMotes can be deployed simultaneously with a $1 \mathrm{~Hz}$ sampling rate, but the system capacity increases by 2600 eMotes for every second added between samples. All measured and reported data falls within accuracy requirements for reporting with both the World Meteorological Organization (WMO) and the National Oceanic and Atmospheric Administration (NOAA). This paper presents the design and validation of the eMote system alongside discussions on the implementation of a large-scale, low-cost sensor network. The eMote represents unprecedented in-situ atmospheric measurement capabilities with the ability to deploy more than 260 times the number of sensing units as the most comparable commercially available dropsonde.
\end{abstract}

\section{Keywords}

Atmospheric Sensing, Airborne, Biomimetic, MF-TDMA

\section{Introduction}

Atmospheric sensing has been performed primarily through indirect methods such as ground-based radar [1] and satellite imaging [2] [3]. More recently, direct in-situ measurement of the atmosphere has become possible through ground stations [4], weather balloons equipped with radiosondes [5], dropsondes [6], and ACARS data [7]. However, these in-situ methods are limited in the data that they can provide and the atmospheric events that they can measure: 
ACARS is limited to the path of commercial flights [7]; weather balloons only provide data for a single point in space at a time [5] [8], and dropsondes weigh too much and fall too fast to be deployed over populated areas [9] [10]. Additionally, an estimated 3\% of the United States' gross domestic product is affected by weather variability [11], indicating that there is a clear need for an accurate, large-scale in-situ measurement solution to improve weather forecasting models.

The recent explosion of Internet of Things (IoT) enabling technologies has made new atmospheric sensing solutions possible [12] [13] [14]. As the IoT concept gained traction, the costs of various sensing technologies and wireless communication solutions dropped significantly [15]. IoT systems are characterized by massively interconnected subsystems that work in tandem to provide new insight. These new ubiquitous sensing systems inspired the design of a massively deployable, low-cost, in-situ atmospheric sensing mote called eMote.

The eMote is designed for mass deployment over atmospheric events of interest as shown in Figure 1. Up to 2080 eMotes can be deployed simultaneously and report the measured air temperature, air pressure, relative humidity, and estimated wind speeds with accompanying GPS coordinates and timestamps. The eMotes are small, lightweight, and autorotate upon release to slow their descent to an average fall speed of $2.5 \mathrm{~m} / \mathrm{s}$ at sea level. A large-scale deployment of eMotes over a storm or area of interest will allow for atmospheric data to be gathered with unprecedented spatial and temporal density. A short comparison of the eMote system with commercially available dropsondes that perform similar measurement functions is shown in Table 1 and indicates that an eMote deployment

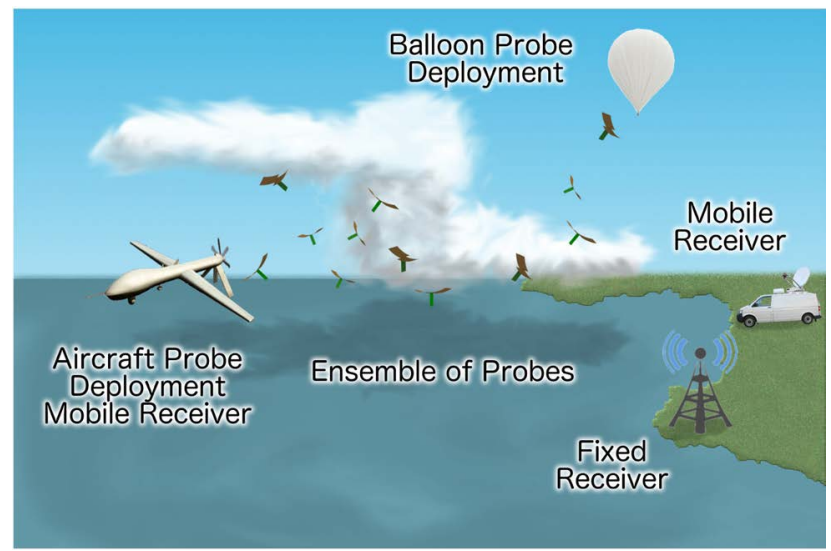

Figure 1. eMote deployment scenario.

Table 1. Comparison of eMote to state of the art in in-situ atmospheric measuring devices.

\begin{tabular}{ccccc}
\hline Device & Range (km) & Max Deployment & Fall Speed (m/s) & Weight (g) \\
\hline eMote & $50($ est.) & 2080 & $<3$ & 12 \\
Vaisala RD94 [6] & 150 & 8 & 11 w/parachute & 350 \\
Vaisala RS41 [16] & 160 & 1 & 11 w/parachute & 113 \\
Qinetiq TASK [17] & 150 & 2 & unknown & 88 \\
\hline
\end{tabular}


could gain orders of magnitude more data and provide new insights into atmospheric phenomena.

This paper discusses the design and verification of the eMote. First, different aspects of the eMote's design are discussed including the physical shape, antenna structure, component selection, and network strategy. Next, validation of the eMote is discussed through both sensor and antenna characterization as well as range testing and network strategy verification. Finally, closing remarks are provided.

\section{2. eMote Design}

Designing individual nodes for a large-scale sensor network brings a unique set of constraints. The power consumption, size, and cost must be minimized while maximizing the accuracy, range, and throughput of the system. Additionally, the eMotes must be able to be safely deployed from a high altitude over populated areas without risk of damaging structures or people below, necessitating an extremely low mass and fall speed [9]. In order to meet all of these application requirements, inspiration was derived from the auto-rotation of maple seeds [18] and components with multiple integrated functions were used. Here, the physical design, antenna design, component selection, and network strategy for the eMote are all discussed in detail.

\subsection{Physical Design}

An image of a fabricated eMote is shown in Figure 2. The unique shape of the printed circuit board serves two purposes: to induce auto-rotation and to aspirate the temperature sensor. This shape closely mimics that of the maple seed, or samara, whose evolution naturally selected an asymmetrical seed shape with a long, thin rotor structure and low, off-center center of mass to minimize fall speed and allow airborne seeds to travel farther [19] [20]. In an effort to follow these principles, the center of mass for the eMote is located far below the flexible rotor/antenna structure in order to induce auto-rotation which slows its descent [21]. The final dimensions of both the flexible rotor/antenna structure and

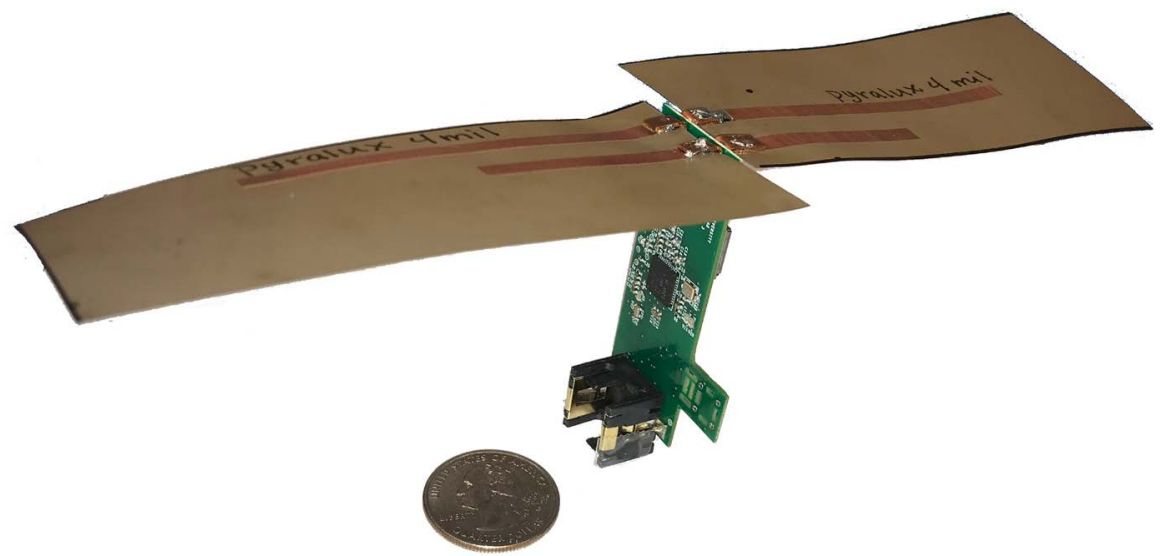

Figure 2. Fabricated eMote with US quarter for scale. 
printed circuit board were chosen after iterative testing and refinement of eMote mockups constructed of FR4 and weighted with washers to match the weight distribution. These eMote mock-ups were dropped from various heights in both indoor and outdoor scenarios to determine the size and shape that maximized rotation and minimized fall speed. Additionally, the temperature sensor resides on the small protrusion with ventilation cutouts in order to aspirate the sensor while the eMote is spinning, increasing the accuracy of temperature measurements by isolating the sensor and minimizing thermal bias induced by other components.

\subsection{Antenna Design}

The eMote antenna structure serves a secondary purpose as a rotor to enable auto-rotation and slow descent. The structure contains two separate antennas: a $915 \mathrm{MHz}$ Region 2 industrial, scientific, and medical (ISM) band antenna for eMote data transmission and a GPS L1 band antenna, each designed with a larger than necessary bandwidth to allow for manufacturing tolerances. The antennas were designed and fabricated onto a flexible 4 mil Pyralux AP substrate to allow the rotors to bend and flex while falling. The final dimensions of the antenna structure can be seen in Figure 3, where the necessary asymmetry for auto-rotation is clearly visible.

\subsection{Component Selection}

Components for the eMote were chosen in an effort to minimize cost, weight, and power consumption. The main components chosen were the Texas Instruments CC430F5137 embedded microcontroller and ISM band transceiver, Measurement Specialties MS5803 air pressure and temperature sensor, Sensirion SHT25 humidity and temperature sensor, and the u-Blox Max M8 GPS receiver. The GPS module communicates with the microcontroller through a low data rate UART connection to provide a $1 \mathrm{~Hz}$ time-stamp alongside GPS coordinates, while the two atmospheric sensors communicate measurements through $\mathrm{I}^{2} \mathrm{C}$. These protocols were chosen in an effort to minimize power consumption through the use of the CC430F5137's low-power modes as discussed in the following subsection. Further, these specific sensors were chosen due to their low cost and acceptable accuracy specifications for data reporting with the World Meteorological Organization (WMO) [22] and National Oceanic and Atmospheric Administration (NOAA) [23]. We estimate that large-scale production of eMotes

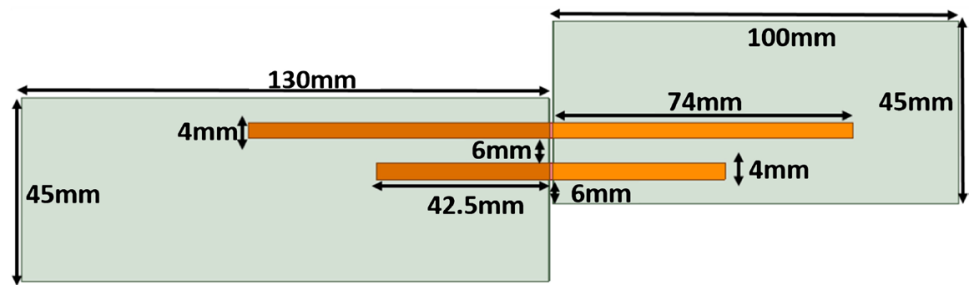

Figure 3. ISM and GPS L1 band antenna/rotor structure dimensions. 
could be achieved at a unit cost of around 100 \$, much less than other in-situ atmospheric sensing solutions [6] [16] [17].

\subsection{Network Strategy}

The eMotes utilize several techniques to reduce power consumption and provide as much data as possible. Onboard the eMotes themselves, UART and $\mathrm{I}^{2} \mathrm{C}$ are used to minimize power consumption by the microcontroller. The UART interface provides time-stamp and location information from the GPS module once every second that is synchronized by the GPS satellites; this single UART communication wakes the microcontroller from an ultra low-power mode and establishes a synchronized time-stamp across all deployed eMotes. In order to collect data each second, a single $\mathrm{I}^{2} \mathrm{C}$ bus is used to communicate with both sensor modules using minimal time in an active mode. The minimization of active peripherals on the eMote reduces power consumption and allows the device to be in ultra low-power modes for more than $50 \%$ of the time when reporting data once per second.

In order to receive coherent data from over 2000 eMotes at once, a Multiple Frequency Time-Division Multiple Access (MF-TDMA) scheme is used. As the on-board GPS module provides a synchronized time-stamp every second, all deployed eMotes are able to accurately select a time slot using a simple timer module. Each eMote is given a unique identification signature when it is programmed and uses this signature to determine a time slot and frequency channel for its transmissions. In practice, the eMotes are able to make use of 130 individual channels containing 16 time slots per second within the $915 \mathrm{MHz}$ ISM band. It is worth noting that each additional second added between transmissions adds 20 time slots per channel, as 4 time slots of the first second have been allocated for GPS reception. These additional time slots will allow for the deployment of 2600 additional eMotes for each second added. Furthermore, over-sized time slots and forward error correction codes were used to reduce interference and maximize the effective range of the eMotes [24].

\section{3. eMote Validation}

All aspects of the eMote have been verified through rigorous testing. In this section test results are presented to validate the accuracy of the reported data, the design and function of the antenna-rotor structure, the maximum range of the system, and the scalability of the MF-TDMA network strategy.

\subsection{Sensor Testing and Characterization}

The eMote's atmospheric sensing capabilities were verified through several test deployments. Figure 4 shows a sample set of data collected from an overnight deployment of 5 eMotes across Auburn University's campus alongside a Davis Instruments Vantage Pro2 weather station to serve as a reference. eMotes have also been tested in several different climes and deployment scenarios: the aforementioned 


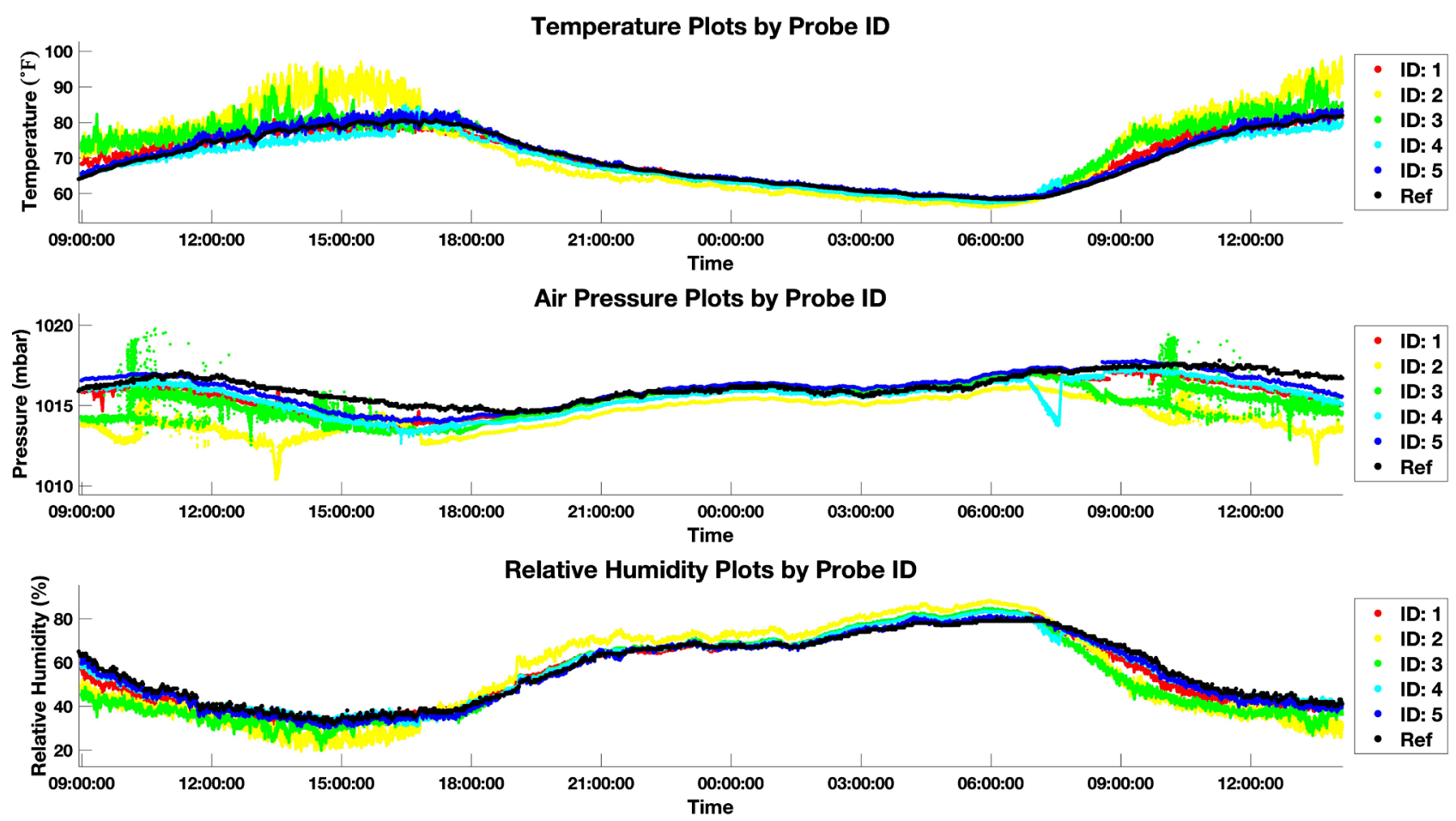

Figure 4. Plot of recorded atmospheric data from 9 am on September 7, 2017 to 2 pm on September 8, 2017.

overnight test deployments; low altitude, small scale deployments from a drone over rural areas near Auburn, AL; and several kilometer high deployments over New Mexico and Alaska testing facilities in conjunction with Sandia National Labs. Data from these tests have been compared to reference data from other atmospheric sensing systems and organizations, such as the Meteorological Terminal Aviation Routine Weather Report (METAR), to calculate the accuracy of reported eMote data. The calculated accuracies for eMote data and accuracy requirements for data reporting with the WMO [22] and NOAA [23] are shown in Table 2.

\subsection{Antenna-Rotor Testing}

Verification of the antenna-rotor structure was carried out in two phases: physical testing of eMote auto-rotation and characterization of the two antennas under different bend conditions.

To test the auto-rotation of the eMote, antenna-rotor structures were attached to eMote mock-ups made from FR4 and weighted with washers to emulate the mass distribution of a fully fabricated eMote. These drop units were then dropped from various altitudes: a $15 \mathrm{~m}$ drop from the roof of a campus building, a $16 \mathrm{~m}$ indoor drop from the rafters of the campus basketball arena, and $50 \mathrm{~m}$ and 100 $\mathrm{m}$ drops from a drone over nearby farmland. These drop tests were later repeated with fully fabricated eMotes. The average fall time of both the drop units and fabricated eMotes was found to be $2.51 \mathrm{~m} / \mathrm{s}$, which is significantly slower than other in-situ atmospheric measurement systems and allows for more data collection [6] [16] [17]. 
Characterization of the ISM and GPS L1 band antennas was performed in an anechoic chamber with a Keysight FieldFox N9951A and Diamond Antenna Measurement Systems DAMS7000. Figure 5 shows the measured and simulated antenna patterns; the antenna-rotor structure has been designed so that the nulls point to the horizon during free-fall, maximizing GPS satellite signal reception from satellites above and eMote data transmission towards a ground station below. Further, the voltage standing wave ratio (VSWR) was measured for different bend angles of the antenna-rotor structure, as shown in Figure 6, to verify

Table 2. Calculated eMote accuracies vs. WMO and NOAA accuracy requirements [22] [23].

\begin{tabular}{cccc}
\hline Measurement & eMote & WMO [22] & NOAA [23] \\
\hline Temperature & $\pm 0.88^{\circ} \mathrm{F}$ & $\pm 3.6^{\circ} \mathrm{F}$ & $\pm 1^{\circ} \mathrm{F}$ \\
Air Pressure & $\pm 0.49 \mathrm{mbar}$ & $\pm 1 \mathrm{mbar}$ & $\pm 0.7 \mathrm{mbar}$ \\
Relative Humidity & $\pm 1.81 \%$ & $\pm 5 \%$ & $\pm 1.5 \%$ \\
Wind Speed (indirect) & N/A & $\pm 1 \mathrm{~m} / \mathrm{s}$ & $\pm 0.51 \mathrm{~m} / \mathrm{s}$ \\
\hline
\end{tabular}

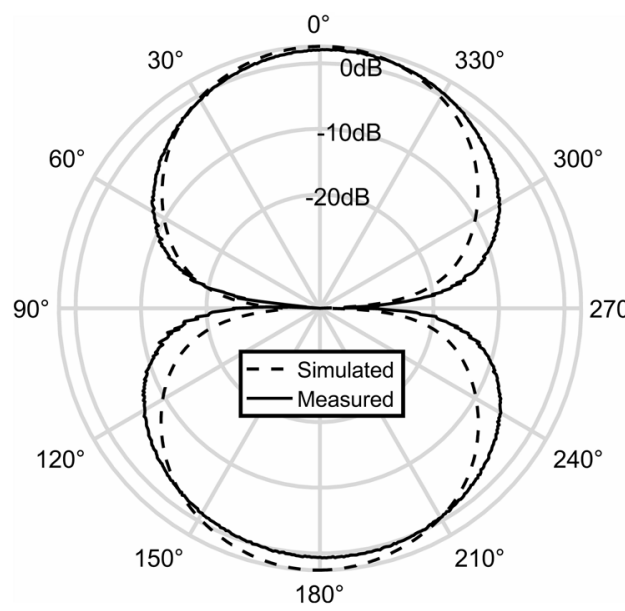

(a)

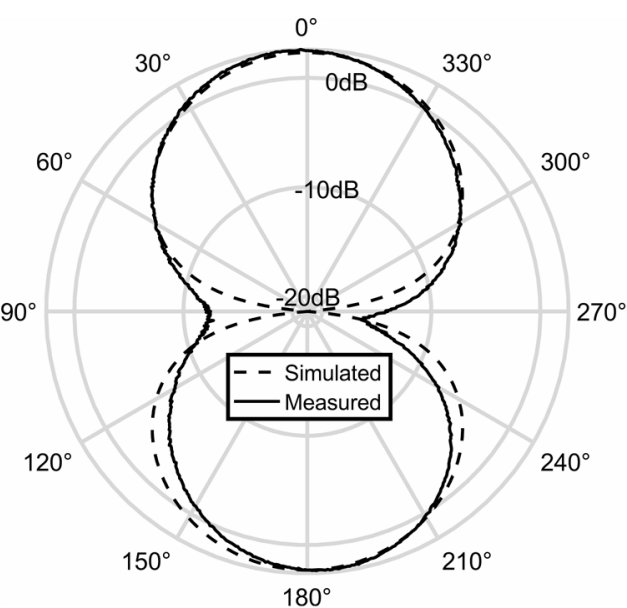

(b)

Figure 5. Directivity in dB of (a) GPS and (b) ISM antennas; patterns are oriented to be broadside at the $0^{\circ}$ position.

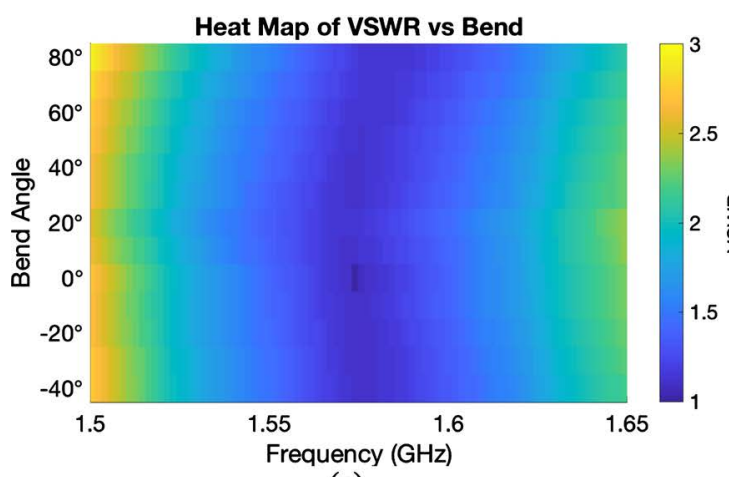

(a)

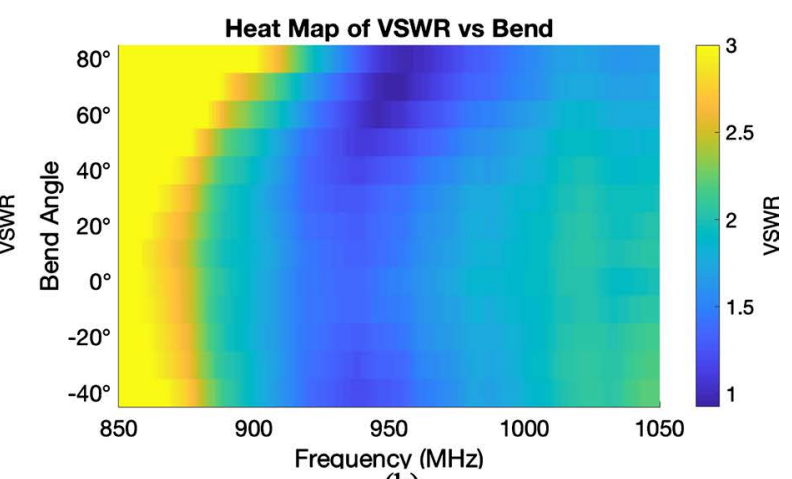

(b)

Figure 6. Measured VSWR during bend testing (a) GPS L1 band and (b) ISM band antennas. 
the designed antennas will still function with a VSWR of less than 2 for the entire ISM and GPS L1 bands for all reasonable bend angles that may occur during free fall.

\subsection{Range Testing}

Long-range eMote operation was verified both in the lab and through actual deployments. In-line attenuators were used inside an anechoic chamber to simulate distance between the eMote and receiver station, yielding a maximum range of roughly $50 \mathrm{~km}$. In order to verify this number in the field, eMotes were attached to balloons and allowed to drift off on the wind. This test was performed several times in different weather conditions to verify long-range operation. The average maximum distance at which valid packets were received for these tests was $14.5 \mathrm{~km}$, with a maximum distance of $18.5 \mathrm{~km}$ as shown in Figure 7. This tested range is on the same order of magnitude as that found in laboratory conditions and could potentially be increased by using a different modulation technique or by adding amplifiers to the receiver base station.

\subsection{Network Strategy Testing}

The scalability of the MF-TDMA strategy adopted for eMotes was tested through several ground-based large-scale deployments of 44, 80, and 83 eMotes. In each test, the eMotes were run for over 30 minutes to provide a large sample size for assessing the viability of the network. In these tests, packets were received from at least $90 \%$ of the deployed eMotes over the course of the test, but only an average of around $60 \%$ of sent packets were received. These low packet reception rates are likely due to multipath and interference issues arising from the placement of the eMotes, as both the eMotes and receivers were placed on or near the

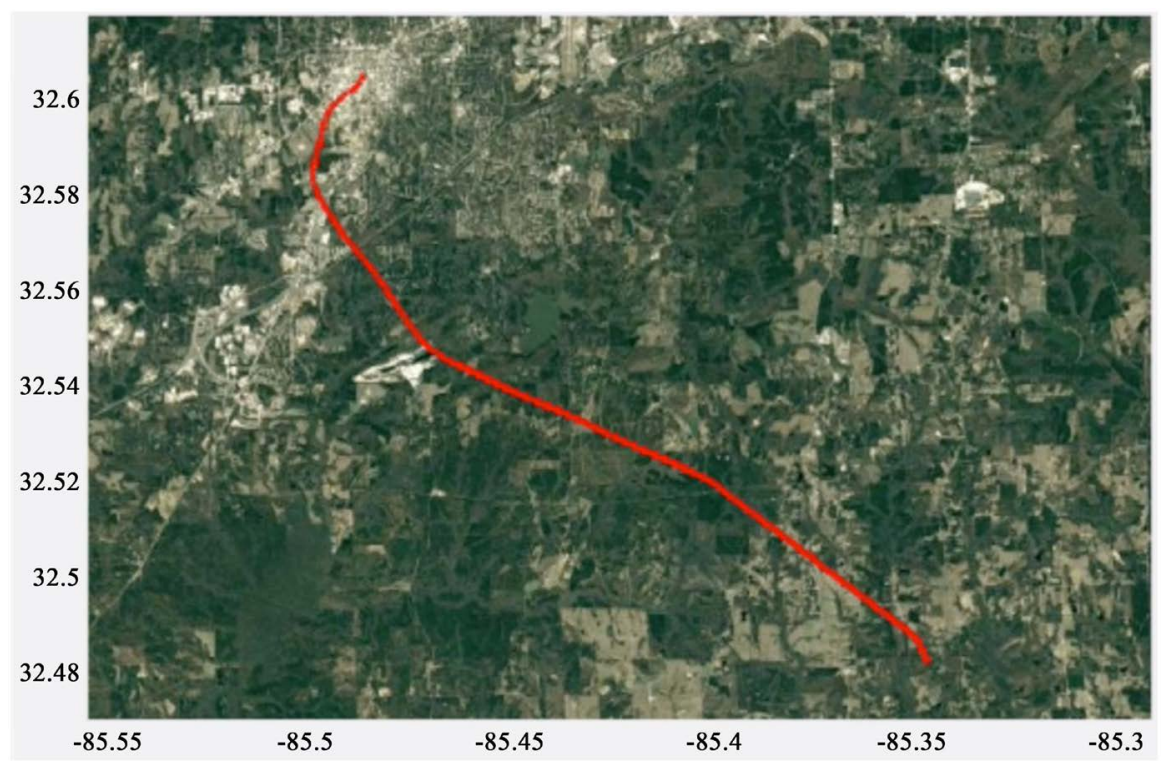

Figure 7. Example received GPS data from long-range outdoor test beginning at $(-85.49$, $32.59)$ and ending near $(-85.35,32.48)$. 


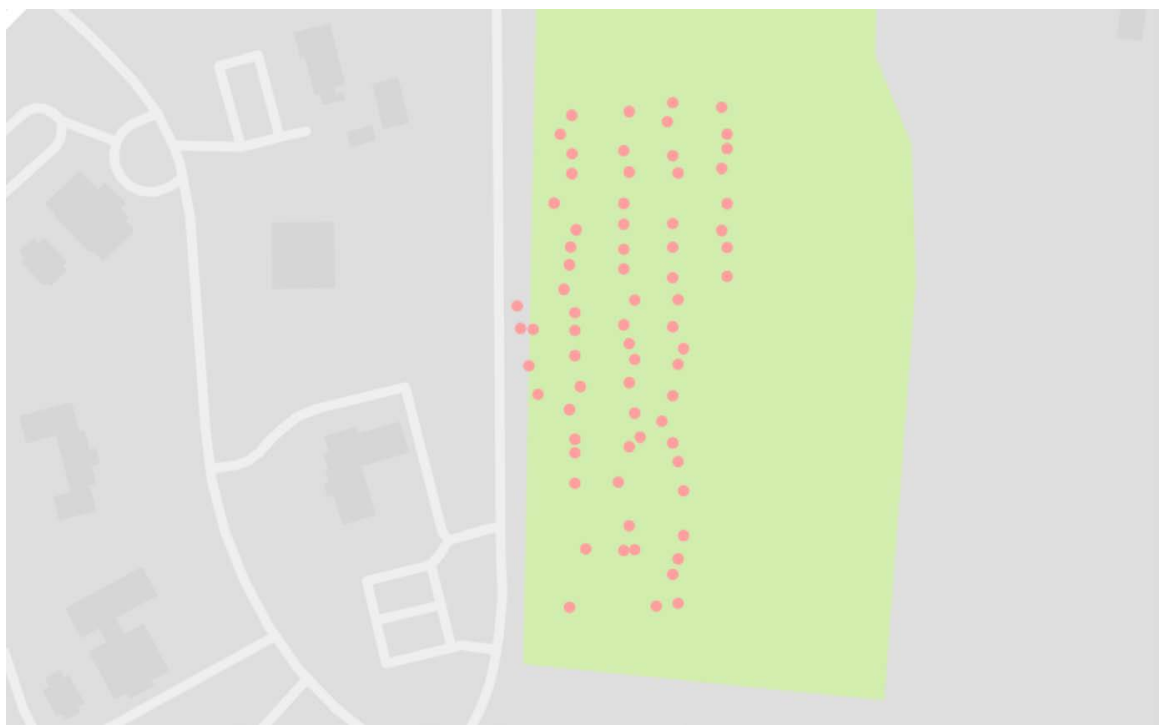

Figure 8. Recorded GPS locations of eMotes during large-scale deployment test.

ground. Figure 8 shows the placement of eMotes in an intramural field on Auburn University's campus during the 83 eMote test.

\section{Conclusion}

A large-scale airborne atmospheric sensor network has been designed, fabricated, and tested in various deployment scenarios. The eMote represents a new level of atmospheric sensing due to its massively deployable nature. This system, inspired by the advent of ubiquitous sensing solutions with the Internet of Things, is able to provide data with greater spatial and temporal density than any current commercially available system. This will allow for real-time monitoring and recording of atmospheric behavior, rather than the vertical profiles provided by comparable airborne probes. The eMote may provide new insights into the development and behavior of large-scale atmospheric events.

\section{Conflicts of Interest}

The authors declare no conflicts of interest regarding the publication of this paper.

\section{References}

[1] Li, N., et al. (2017) The Assessment of Ground-Based Weather Radar Data by Comparison with TRMM PR. IEEE Geoscience and Remote Sensing Letters, 14, 72-76. https://doi.org/10.1109/LGRS.2016.2626320

[2] Durden S.L. and Perkovic-Martin, D. (2017) The RapidScat Ocean Winds Scatterometer: A Radar System Engineering Perspective. IEEE Geoscience and Remote Sensing Letters, 5, 36-43. https://doi.org/10.1109/MGRS.2017.2678999

[3] Veefkind, J., et al. (2012) TROPOMI on the ESA Sentinel-5 Precursor: A GMES Mission for Global Observations of the Atmospheric Composition for Climate, Air Quality and Ozone Layer Applications. Remote Sensing of Environment, 120, 70-83. https://doi.org/10.1016/j.rse.2011.09.027 
[4] Zorer, R., et al. (2013) Daily MODIS Land Surface Temperature Data for the Analysis of the Heat Requirements of Grapevine Varieties. IEEE Transactions on Geoscience and Remote Sensing, 51, 2128-2135. https://doi.org/10.1109/TGRS.2012.2226465

[5] Galvin, J.F.P. (2003) Back to Basics: Radiosondes: Part 2-Using and Interpreting the Data. Weather, 58, 387-395. https://doi.org/10.1256/wea.126.02B

[6] Vaisala (2010) Vaisala Dropsonde RD94. https://www.vaisala.com/sites/default/files/documents/RD94-Datasheet-B210936EN -B.pdf

[7] Moninger, W.R., Mamrosh, R.D. and Pauley, P.M. (2003) Automated Meteorological Reports from Commercial Aircraft. Bulletin of the American Meteorological Society, 84, 203-216. https://doi.org/10.1175/BAMS-84-2-203

[8] Liu, Z., Wong, M.S., Nichol, J. and Chan, P.W. (2013) A Multi-Sensor Study of Water Vapour from Radiosonde, MODIS and AERONET: A Case Study of Hong Kong. International Journal of Climatology, 33, 109-120. https://doi.org/10.1002/joc.3412

[9] Xinhua, F., Jun, S., Beiguo, L. and Yonggang, T. (2013) Design and Implementation of Dropsonde Wind Measurement System, 2013 IEEE 11th International Conference on Electronic Measurement Instruments, Harbin, 16-19 August 2013, 166-169. https://doi.org/10.1109/ICEMI.2013.6743007

[10] Busen, R. (2000) The Release of Dropsondes: A Hazard for Commercial Air Traffic? Air Traffic Control Quarterly, 8, 155-171. https://doi.org/10.2514/atcq.8.2.155

[11] Lazo, J.K., Lawson, M., Larsen, P.H. and Waldman, D.M. (2011) U.S. Economic Sensitivity to Weather Variability. Bulletin of the American Meteorological Society, 92, 709-720. https://doi.org/10.1175/2011BAMS2928.1

[12] Xu, B., Zheng, J. and Wang, Q. (2016) Analysis and Design of Real-Time Micro-Environmental Parameter Monitoring System Based on Internet of Things. 2016 International Conference on Internet of Things (iThings) and IEEE Green Computing and Communications (GreenCom) and IEEE Cyber, Physical and Social Computing (CPSCom) and IEEE Smart Data (SmartData), Chengdu, 15-18 December 2016, 368-371. https://doi.org/10.1109/iThings-GreenCom-CPSCom-SmartData.2016.87

[13] Ahmed, M.M., Banu, S. and Paul, B. (2017) Real-Time Air Quality Monitoring System for Bangladesh's Perspective Based on Internet of Things. 2017 3rd International Conference on Electrical Information and Communication Technology (EICT), Khulna, 7-9 December 2017, 1-5. https://doi.org/10.1109/EICT.2017.8275161

[14] Mukherji, S.V., Sinha, R., Basak, S. and Kar, S.P. (2019) Smart Agriculture Using Internet of Things and MQTT Protocol. 2019 International Conference on Machine Learning, Big Data, Cloud and Parallel Computing (COMITCon), Faridabad, 14-16 February 2019, 14-16. https://doi.org/10.1109/COMITCon.2019.8862233

[15] Mania, F., Santos, C.H.S. and Alvaro, A. (2014) Outlining Low Costs and Open Embedded Systems for RFID in Internet of Things Applications. 2014 IEEE Brasil RFID, Sao Paulo, 25 September 2014, 16-18.

https://doi.org/10.1109/BrasilRFID.2014.7128954

[16] Vaisala. (2018) Vaisala Radiosonde RS41-D. https://www.vaisala.com/en/products/instruments-sensors-and-other-measurement -devices/soundings-products/rs41

[17] Qinetiq (2015) TASK: Tactical Atmospheric Sounding Kit. https://qinetiq-na.com/products/metsense/task/ 
[18] Pounds, P., et al. (2016) Automatic Distribution of Disposable Self-Deploying Sensor Modules. Experimental Robotics, 109, 535-543.

https://doi.org/10.1007/978-3-319-23778-7 35

[19] Stevenson, R.A., Evangelista, D. and Looy, C.V. (2015) When Conifers Took Flight: A Biomechanical Evaluation of an Imperfect Evolutionary Takeoff. Paleobiology, 41, 205-225. https://doi.org/10.1017/pab.2014.18

[20] Norberg, R.A. (1973) Autorotation, Self-Stability, and Structure of Single-Winged Fruits and Seeds (Samaras) with Comparative Remark on Animal Flight. Biological Reviews, 48, 561-596. https://doi.org/10.1111/j.1469-185X.1973.tb01569.x

[21] Lentik, D., Dickson, W.B., Van Leeuwen, J.L. and Dickinson, M.H. (2009) Leading-Edge Vortices Elevate Lift of Autorotating Plant Seeds. Science, 324, 1438-1440. https://doi.org/10.1126/science.1174196

[22] World Meteorological Organization (2017) Observing Systems Capability Analysis and Review Tool. https://www.wmo-sat.info/oscar/requirements

[23] National Oceanic and Atmospheric Administration (2017) NWS Directives System. http://www.nws.noaa.gov/directives/010/010.php

[24] Hoel, R. (2007) Design Note DN504: FEC Implementation. http://www.ti.com/lit/an/swra113a/swra113a.pdf 